\title{
A New Hybrid Iterative Method for Solving Fixed Points Problems for a Finite Family of Multivalued Strictly Pseudo-Contractive Mappings and Convex Minimization Problems in Real Hilbert Spaces
}

\author{
Thierno M. M. Sow
}

\begin{abstract}
In this paper, we investigate the problem of finding a common solution to fixed point problem involving a finite family of multivalued strictly pseudo-contractive mappings and convex minimization problem in the framework of Hilbert spaces. Inspired by the proximal point algorithm and general iterative method, a new iterative method for solving the problem is introduced. Strong convergence theorem of the proposed method is established without any compactness assumption. Our scheme generalize and extend some of the existing results in the literature.
\end{abstract}

Keywords: Fixed points problems, Convex minimization problem, Set-valued operators, Iterative methods

AMS Subject Classification (2020): Primary: 47H09; Secondary: 49J20; $49 J 40$

\section{Introduction}

Let $H$ be a real Hilbert space with the inner product $\langle.,$.$\rangle and norm \|$.$\| respectively. Let K$ be a nonempty closed convex subset of $H$. Consider the following convex minimization problem: find $x \in K$ such that

$$
g(x)=\min _{y \in K} g(y)
$$

where $g: H \rightarrow(-\infty,+\infty)$ be a proper convex and lower semi-continuous. The set of all minimizers of $g$ on $K$ is denoted by $\operatorname{argmin}_{y \in K} g(y)$. In 1970, Martinet [21] introduced and studied the proximal point algorithm (PPA) for solving optimization problems. Thereafter the likes of Rockafellar [29], find a solution of the constrained convex minimization problem in the frame work of Hilbert space by using PPA. Let $g$ be a proper convex and lower 
semi-continuous function on $\mathrm{H}$. The PPA is defined as

$$
\left\{\begin{array}{l}
x_{1} \in H, \\
x_{n+1}=\operatorname{argmin}_{y \in H}\left[g(y)+\frac{1}{2 \lambda_{n}}\left\|x_{n}-y\right\|^{2}\right],
\end{array}\right.
$$

where $\lambda_{n}>0$ for all $n \geq 1$. It was proved that the sequence $\left\{x_{n}\right\}$ converges weakly to a minimizer of $g$ provided $\sum_{n=0}^{\infty} \lambda_{n}=\infty$. In [12], it was shown that a PPA does not necessarily converges strongly. The fact that a PPA does not necessarily converges strongly have been overcome by researchers in this area by introducing a more general PPA in different spaces to obtain a weak and strong convergence. Over the years, researcher have been able to further extend the convex minimization problems by finding a common element of the set of solutions of various convex minimization problems and the set of fixed points for nonexpansive mappings in Hilbert spaces and Banach spaces ( see, e.g., Güler [12], Solodov and Svaiter [31], Kamimura and Takahashi [14], Lehdili and Moudafi [15], Reich, [28], Chidume and Djitte [7, 8] and the references therein).

Let $(X, d)$ be a metric space, $K$ be a nonempty subset of $X$ and $T: K \rightarrow 2^{K}$ be a multivalued mapping. An element $x \in K$ is called a fixed point of $T$ if $x \in T x$. For single valued mapping, this reduces to $T x=x$. The fixed point set of $T$ is denoted by $F(T):=\{x \in D(T): x \in T x\}$.

The fixed point theory of multi-valued mappings is much more complicated and harder than the corresponding theory of single-valued mappings. However, some classical fixed point theorems for single-valued mappings have already been extended to multi-valued mappings; (see, for example, Brouwer [4], Kakutani [13], Nash [24, 25], Garcia-Falset et al. [27]). The recent fixed point results for multi-valued mappings can be found Blasi et al. [3], Sow [32], Sene et al. [30], Sow et al. [30] and the references cited therein.

Interest in the study of fixed point theory for multi-valued nonlinear mappings stems, perhaps, mainly from its usefulness in real-world applications such as Game Theory and Non-Smooth Differential Equations, Optimization.

Let $D$ be a nonempty subset of a normed space $E$. The set $D$ is called proximinal (see, e.g., [26]) if for each $x \in E$, there exists $u \in D$ such that

$$
d(x, u)=\inf \{\|x-y\|: y \in D\}=d(x, D),
$$

where $d(x, y)=\|x-y\|$ for all $x, y \in E$. Every nonempty, closed and convex subset of a real Hilbert space is proximinal. Let $C B(D), K(D)$ and $P(D)$ denote the family of nonempty closed bounded subsets, nonempty compact subsets, and nonempty proximinal bounded subsets of $D$ respectively. The Pompeiu Hausdorff metric on $C B(D)$ is defined by:

$$
H(A, B)=\max \left\{\sup _{a \in A} d(a, B), \sup _{b \in B} d(b, A)\right\}
$$

for all $A, B \in C B(D)$ (see, Berinde [2]). A multi-valued mapping $T: D(T) \subseteq E \rightarrow C B(E)$ is called L-Lipschitzian if there exists $L>0$ such that

$$
H(T x, T y) \leq L\|x-y\|, \quad \forall x, y \in D(T) .
$$

When $L \in(0,1)$, we say that $T$ is a contraction, and $T$ is called nonexpansive if $L=1$.

A mapping $A: K \rightarrow H$ is said to be $k$-strongly monotone if there exists $k \in(0,1)$ such that for all $x, y \in K$,

$$
\langle A x-A y, x-y\rangle_{H} \geq k\|x-y\|^{2} .
$$

A mapping $A: K \rightarrow H$ is said to be strongly positive bounded linear if there exists a constant $k>0$ such that

$$
\langle A x, x\rangle_{H} \geq k\|x\|^{2}, \quad \forall x \in K .
$$

Remark 1.1. From the definition of $A$, we note that strongly positive bounded linear operator $A$ is a $\|A\|$-Lipschitzian and $k$-strongly monotone operator. 
Great attention has been paid to single-valued nonexpansive mappings (a special kind of strictly pseudo-contractive mappings) because many nonlinear problems can be reduced to fixed point problems of nonexpansive mappings. Among these iterative methods, the Mann iteration method is the mostfavour fixed point algorithm for nonexpansive mappings since many algorithms can be reducedto Mann iteration. Recall that Mann's iteration process [16] is defined as follows: Let $C$ be a nonempty, closed and convex subset of a Banach space $X$, Mann's scheme is defined by

$$
\left\{\begin{array}{l}
x_{0} \in C, \\
x_{n+1}=\alpha_{n} x_{n}+\left(1-\alpha_{n}\right) T x_{n},
\end{array}\right.
$$

$\left\{\alpha_{n}\right\}$ is a sequence in $(0,1)$. But Mann's iteration process has only weak convergence, even in Hilbert space setting. Therefore, many authors try to modify Mann's iteration to have strong convergence for nonlinear operators (see, e.g., [33], [30]).

In 2009, Yao et al. motivated by the fact that Mann's algorithm method is remarkably useful for finding fixed points of a nonexpansive mapping, they proved the following theorem.

Theorem 1.1. [37] Let $H$ be a real Hilbert space. Let $T: H \rightarrow H$ be a nonexpansive mapping with $F(T) \neq \emptyset$. For given $x_{0} \in H$, let the sequences $\left\{x_{n}\right\}$ and $\left\{y_{n}\right\}$ be generated iteratively by

$$
\left\{\begin{array}{l}
y_{n}=\left(1-\alpha_{n}\right) x_{n} \\
x_{n+1}=\beta_{n} y_{n}+\left(1-\beta_{n}\right) T y_{n},
\end{array}\right.
$$

$\left\{\beta_{n}\right\}$ and $\left\{\alpha_{n}\right\}$ are a real sequences in $(0,1)$ satisfying:

(i) $\lim _{n \rightarrow \infty} \alpha_{n}=0 ; \quad$ (ii) $\sum_{n=0}^{\infty} \alpha_{n}=\infty$.

Then the sequences $\left\{x_{n}\right\}$ and $\left\{y_{n}\right\}$ generated by (1.4) converge strongly to fixed point of $T$.

Recently, iterative methods for single-valued nonexpansive mappings have been applied to solve fixed points problems and variational inequality problems in Hilbert spaces, see, e.g.,[18, 19, 35] and the references therein.

A typical problem is to minimize a quadratic function over the set of the fixed points of a nonexpansive mapping on a real Hilbert space H:

$$
\min _{x \in F(T)} \frac{1}{2}\langle A x, x\rangle-\langle b, x\rangle .
$$

In [35], Xu proved that the sequence $\left\{x_{n}\right\}$ defined by iterative method below with initial guess $x_{0} \in H$ chosen arbitrary:

$$
x_{n+1}=\alpha_{n} b+\left(I-\alpha_{n} A\right) T x_{n}, \quad n \geq 0,
$$

converges strongly to the unique solution of the minimization problem (1.5), where $T$ is a nonexpansive mappings in $H$ and $A$ a strongly positive bounded linear operator. In 2006 Marino and Xu [18] extended Moudafi's results [20] and Xu's results [35] via the following general iteration $x_{0} \in H$ and

$$
x_{n+1}=\alpha_{n} \gamma f\left(x_{n}\right)+\left(I-\alpha_{n} A\right) T x_{n}, n \geq 0,
$$

where $\left\{\alpha_{n}\right\}_{n \in \mathbb{N}} \subset(0,1), A$ is bounded linear operator on $H$ and $T$ is a nonexpansive. Under suitable conditions, they proved the sequence $\left\{x_{n}\right\}$ defined by (1.7) converges strongly to the fixed point of $T$, which is a unique solution of the following variational inequality

$$
\left\langle A x^{*}-\gamma f\left(x^{*}\right), x^{*}-p\right\rangle \leq 0, \quad \forall p \in F(T) .
$$

The important class of single-valued $k$-strictly pseudo-contractive maps on Hilbert spaces was introduced by Browder and Petryshyn [5] as a generalization of the class of nonexpansive mappings.

Definition 1.1. Let $K$ be a nonempty subset of a real Hilbert space $H$. A map $T: K \rightarrow H$ is called k-strictly pseudo-contractive if there exists $k \in(0,1)$ such that

$$
\|T x-T y\|^{2} \leq\|x-y\|^{2}+k\|x-y-(T x-T y)\|^{2}, \quad \forall x, y \in K .
$$


It is trivial to see that every nonexpansive map is strictly pseudo-contractive. Motivated by this, Chidume et al. [10] introduced the of multivalued strictly pseudo-contractive mappings in real Hilbert as follows.

Definition 1.2. A multi-valued mapping $T: D(T) \subseteq H \rightarrow C B(H)$ is said to be $k$-strictly pseudo-contractive, if there exists $k \in(0,1)$ such for all $x, y \in D(T)$, we have

$$
(H(T x, T y))^{2} \leq\|x-y\|^{2}+k\|(x-u)-(y-v)\|^{2}, \forall u \in T x, v \in T y .
$$

if $k=1$ in (1.9), the map $T$ is said to be pseudo-contractive.

Remark 1.2. It is easily seen that any multivalued nonexpansive mapping is k-strictly pseudocontractive for any $k \in(0,1)$. Moreover the inverse is not true (see,e.g., Sene et al. [30]).

With this definition at hand, many mathematicians proved some strong convergence theorems for approximating fixed points of multivalued k-strictly pseudo-contrcative mappings under some compactness conditions (see, for example, Sene et al. [30], Chidume et .al [10], Sow et al. [34] ).

In 2019, A. A. Mebawondu [22] introduced the following iterative method to find a common element of the set of minimizers of a convex function and the set of common fixed points of a finite family of multivalued nonexpansive mappings, proved the following theorem.

Theorem 1.2 (A. A. Mebawondu [22] ). Let $K$ be a nonempty closed convex subset of a real Hilbert space $H$. Let $m \geq 1$ be a fixed number, for $i, 1 \leq i \leq m$, let $T_{i}: K \rightarrow C B(K)$ be a multivalued nonexpansive mappings and $f: K \rightarrow(-\infty,+\infty)$ be a proper convex and lower semi-continuous function such that $\Gamma:=\bigcap_{i=1}^{m} F\left(T_{i}\right) \cap \operatorname{argmin}_{y \in K} f(y) \neq \emptyset$ and $T_{i} p=\{p\}$ for all $p \in \bigcap_{i=1}^{m} F\left(T_{i}\right)$. Let $\left\{x_{n}\right\}$ be a sequence defined iteratively from arbitrary $x_{1} \in K$ by:

$$
\left\{\begin{array}{l}
y_{n}=J_{\lambda_{n}}^{f} x_{n}, \\
z_{n}=\gamma_{n}{ }^{0} x_{n}+\sum_{i=1}^{m} \gamma_{n}{ }^{i} y_{n}^{i}, \quad v_{n}^{i} \in T_{i} u_{n} \\
x_{n+1}=\alpha_{n}{ }^{0} z_{n}+\left(1-\alpha_{n}{ }^{0}\right) w_{n}, \quad w_{n} \in T_{i} z_{n}
\end{array}\right.
$$

where $=\alpha_{n}{ }^{0} \subset(0,1), \gamma_{n}{ }^{0} \subset(0,1)$ and $\left.\left\{\lambda_{n}\right\} \subset\right] 0, \infty[$ satisfy:

(i) $\sum_{n=0}^{\infty} \alpha_{n}{ }^{0}=\sum_{n=0}^{\infty} \gamma_{n}{ }^{0}=1,($ ii $)\left\{\lambda_{n}\right\}$ is a sequence such that $\lambda_{n} \geq \lambda>0$ for all $n \geq 1$ and some $\lambda$. Then, the sequence $\left\{x_{n}\right\}$ generated by (3.3) converges weakly to an element of $\Gamma$.

In the recent years, the problem of finding a common element of the set of solutions of convex minimization and fixed point problems in real Hilbert spaces have been intensively studied by many authors; see, for example, $[10,16,18,34 ?, 35]$ and the references therein.

In this paper, motivated by above results, the fact that the class of multivalued strictly pseudo-contractive mappings contains those of multivalued nonexpansive and multivalued firmly nonexpansive mappings as subclasses and general proximal point algorithm is remarkably useful for solving most important problems with nonlinear operators, we construct and study an explicit iterative method and prove strong convergence theorems by using a modified general proximal point algorithm for approximating for approximating a common element of the set of minimizers of a convex function and the set of common fixed points of a finite family of multivalued strictly pseudo-contractive mappings in the setting of a real Hilbert space which is a solution of some variational inequalities problems. Our result extends and improves the results of A. A. Mebawondu [22], Yao et al. [37], Marino and $\mathrm{Xu}$ [18] Rockafellar [29] and many other authors.

\section{Preliminaries}

Let us recall the following definitions and results which will be used in the sequel. 
Let $H$ be a real Hilbert space. Let $\left\{x_{n}\right\}$ be a sequence in $H$, and let $x \in H$. Weak convergence of $x_{n}$ to $x$ is denoted by $x_{n} \rightarrow x$ and strong convergence by $x_{n} \rightarrow x$. Let $K$ be a nonempty, closed convex subset of $H$. The nearest point projection from $H$ to $K$, denoted by $P_{K}$ assigns to each $x \in H$ the unique $P_{K} x$ with the property

$$
\left\|x-P_{K} x\right\| \leq\|y-x\|
$$

for all $y \in K$. It is well know that $P_{K}$ satisfies

$$
\left\langle x-P_{K} x, y-P_{K} x\right\rangle \leq 0
$$

for all $y \in K$.

Definition 2.1. Let $H$ be a real Hilbert space and $T: D(T) \subset H \rightarrow 2^{H}$ be a multivalued mapping. $I-T$ is said to be demiclosed at 0 if for any sequence $\left\{x_{n}\right\} \subset D(T)$ such that $\left\{x_{n}\right\}$ converges weakly to $p$ and $d\left(x_{n}, T x_{n}\right)$ converges to zero, then $p \in T p$.

Lemma 2.1 (Demiclosedness Principle, [4]). Let $H$ be a real Hilbert space, $K$ be a nonempty closed and convex subset of $H$. Let $T: K \rightarrow C B(K)$ be a multivalued nonexpansive mapping with convex-values. Then $I-T$ is demi-closed at zero.

Lemma 2.2 ([6]). Let $H$ be a real Hilbert space. Then for any $x, y \in H$, the following inequality hold:

$$
\|x+y\|^{2} \leq\|x\|^{2}+2\langle y, x+y\rangle
$$

Lemma $2.3(\mathrm{Xu},[36])$. Assume that $\left\{a_{n}\right\}$ is a sequence of nonnegative real numbers such that $a_{n+1} \leq\left(1-\alpha_{n}\right) a_{n}+\alpha_{n} \sigma_{n}$ for all $n \geq 0$, where $\left\{\alpha_{n}\right\}$ is a sequence in $(0,1)$ and $\left\{\sigma_{n}\right\}$ is a sequence in $\mathbb{R}$ such that

(a) $\sum_{n=0}^{\infty} \alpha_{n}=\infty$, (b) $\limsup _{n \rightarrow \infty} \sigma_{n} \leq 0$ or $\sum_{n=0}^{\infty}\left|\sigma_{n} \alpha_{n}\right|<\infty$. Then $\lim _{n \rightarrow \infty} a_{n}=0$.

Lemma 2.4. [17] Let $t_{n}$ be a sequence of real numbers that does not decrease at infinity in a sense that there exists a subsequence $t_{n_{i}}$ of $t_{n}$ such that $t_{n_{i}}$ such that $t_{n_{i}} \leq t_{n_{i+1}}$ for all $i \geq 0$. For sufficiently large numbers $n \in \mathbb{N}$, an integer sequence $\{\tau(n)\}$ is defined as follows:

$$
\tau(n)=\max \left\{k \leq n: t_{k} \leq t_{k+1}\right\} .
$$

Then, $\tau(n) \rightarrow \infty$ as $n \rightarrow \infty$ and

$$
\max \left\{t_{\tau(n)}, t_{n}\right\} \leq t_{\tau(n)+1} .
$$

Lemma 2.5. [19] Let $K$ be a nonempty closed convex subset of a real Hilbert space $H$ and $T: K \rightarrow K$ be a mapping.

(i) If $T$ is a $k$-strictly pseudo-contractive mapping, then $T$ satisfies the Lipschitzian condition

$$
\|T x-T y\| \leq \frac{1+k}{1-k}\|x-y\| .
$$

(ii) If $T$ is a k-strictly pseudo-contractive mapping, then the mapping $I-T$ is demiclosed at 0.

Lemma 2.6. [38] Let $H$ be a real Hilbert space. Let $K$ be a nonempty, closed convex subset of $H$ and $A: K \rightarrow H$ be a $k$-strongly monotone and L-Lipschitzian operator with $k>0, L>0$. Assume that $0<\eta<\frac{2 k}{L^{2}}$ and $\tau=\eta\left(k-\frac{L^{2} \eta}{2}\right)$. Then for each $t \in\left(0, \min \left\{1, \frac{1}{\tau}\right\}\right)$, we have

$$
\|(I-t \eta A) x-(I-t \eta A) y\| \leq(1-t \tau)\|x-y\|, x, y \in K .
$$

Lemma 2.7 (Sene et al. [30]). Let $K$ be a nonempty, closed and convex subset of a real Hilbert space $H$ and $\left.\beta_{i} \in\right] 0,1[, i=$ $1, \cdots, n$ such that $\sum_{i=1}^{n} \beta_{i}=1$. Then,

$$
\left\|\sum_{i=1}^{n} \beta_{i} u_{i}\right\|^{2}=\sum_{i=1}^{n} \beta_{i}\left\|u_{i}\right\|^{2}-\sum_{i<j} \beta_{i} \lambda_{j}\left\|u_{i}-u_{j}\right\|^{2} \quad \forall u_{1}, u_{2}, \cdots, u_{n} \in K .
$$


Let $g: K \rightarrow(-\infty,+\infty)$ be a proper convex and lower semi-continuous function. For any $\lambda>0$, define the Moreau-Yosida resolvent of $g$ in a real Hilbert space $H$ as follows:

$$
J_{\lambda}^{g} x=\operatorname{argmin}_{u \in K}\left[g(u)+\frac{1}{2 \lambda}\|x-u\|^{2}\right],
$$

for all $x \in H$. It was shown in [12] that the set of fixed points of the resolvent associated with $g$ coincides with the set of minimizers of $g$. Also, the resolvent $J_{\lambda}^{g}$ of $g$ is nonexpansive for all $\lambda>0$ (see [11]).

Lemma 2.8. (Miyadera [23]) For any $r>0$ and $\mu>0$, the following holds:

$$
J_{r}^{g} x=J_{\mu}^{g} x\left(\frac{\mu}{r} x+\left(1-\frac{\mu}{r}\right) J_{r}^{g} x\right) .
$$

Lemma 2.9 (Sub-differential inequality, [1]). Let $g: H \rightarrow(-\infty,+\infty)$ be a proper convex and lower semicontinuous function. Then, for all $x, y \in H$ and $\lambda>0$, the following sub-differential inequality holds:

$$
\frac{1}{\lambda}\left\|J_{\lambda}^{g} x-y\right\|^{2}-\frac{1}{\lambda}\|x-y\|^{2}+\frac{1}{\lambda}\left\|x-J_{\lambda}^{g} x\right\|^{2}+g\left(J_{\lambda}^{g} x\right) \leq g(y) .
$$

\section{Main Results}

Throughout this section, we will assume that $H$ be a real Hilbert space and $K$ be a nonempty, closed convex subset of $H$. Let $A: K \rightarrow H$ be an $\alpha$-strongly monotone and $L$-Lipschitzian operator, $m \geq 1$ be a fixed number, for $i, 1 \leq i \leq m$, let $T_{i}: K \rightarrow C B(K)$ be a multivalued $k_{i}$-strictly pseudo-contractive mapping and $g: K \rightarrow(-\infty,+\infty)$ be a proper convex and lower semi-continuous function such that $\Gamma:=\bigcap_{i=1}^{m} F\left(T_{i}\right) \cap \operatorname{argmin}_{y \in K} g(y) \neq \emptyset$.

We consider the following fixed point problem:

\section{Problem 1.}

$$
\text { find } x \in K \text { such that } x \in \bigcap_{i=1}^{m} F\left(T_{i}\right) \text {. }
$$

We consider the following convex minimization problem:

\section{Problem 2.}

$$
\text { find } x \in K \text { such that } g(x) \leq g(y), \forall y \in K \text {. }
$$

Remark 3.1. We can observe that $x^{*}$ solves Problem 3.1 and Problem 3.2 if and only if $x^{*} \in \Gamma$.

We show the main result of this paper, that is, the strong convergence analysis for Algorithm 1.

Algorithm 1. Step 0. Take $\left\{\alpha_{n}\right\} \subset(0,1), \eta>0$, and $\left.\left\{\lambda_{n}\right\} \subset\right] 0, \infty\left[\right.$ arbitrarily choose $x_{0} \in K$; and let $n:=0$.

Step 1. Given $x_{n} \in K$, compute $x_{n+1} \in K$ as

$$
\left\{\begin{array}{l}
u_{n}=\operatorname{argmin}_{u \in K}\left[g(u)+\frac{1}{2 \lambda_{n}}\left\|u-x_{n}\right\|^{2}\right], \\
y_{n}=\beta_{0} u_{n}+\sum_{i=1}^{m} \beta_{i} v_{n}^{i}, \quad v_{n}^{i} \in T_{i} u_{n} \\
x_{n+1}=P_{K}\left(I-\alpha_{n} \eta A\right) y_{n}, \quad n \geq 0 .
\end{array}\right.
$$

Update $n:=n+1$ and go to Step 1 .

Where $\left.\beta_{0} \in\right] \mu, 1\left[, \quad \mu:=\max \left\{k_{i}, i=1, \ldots, m\right\}, \quad \beta_{i} \in\right] 0,1\left[\right.$ and $\beta_{0}+\beta_{1}+\cdots+\beta_{m}=1$.

Theorem 3.1. Assume that $I-T_{i}$ is demiclosed at origin and $T_{i} p=\{p\}$ for all $p \in \Gamma$. Suppose that:

(i) $\lim _{n \rightarrow \infty} \alpha_{n}=0 ; \quad($ ii $) 0<\eta<\frac{2 \alpha}{L^{2}}$, and $\sum_{n=0}^{\infty} \alpha_{n}=\infty$ and $\left\{\lambda_{n}\right\}$ is a sequence such that $\lambda_{n} \geq \lambda>0$ for all $n \geq 0$ and some $\lambda$. Then, the sequences $\left\{x_{n}\right\}$ and $\left\{u_{n}\right\}$ defined by Algorithm 1 converge strongly to $x^{*} \in \Gamma$, which is a unique solution of the following variational inequality:

$$
\left\langle A x^{*}, x^{*}-p\right\rangle \leq 0, \quad \forall p \in \Gamma .
$$


Proof. From the choice of $\eta$, properties of $P_{\Gamma}$, and $A$ is strongly monotone, then the variational inequality (3.4) has a unique solution in $\Gamma$. Without loss of generality, we can assume $\alpha_{n} \in\left(0, \min \left\{1, \frac{1}{\tau}\right\}\right)$ where $\tau=\eta\left(k-\frac{L^{2} \eta}{2}\right)$. In what follows, we denote $x^{*}$ to be the unique solution of (3.4). Now, we prove that the sequences $\left\{x_{n}\right\}$ is bounded. Let $p \in \Gamma$. Then, $g(p) \leq g(u)$ for all $u \in K$ This implies that

$$
g(p)+\frac{1}{2 \lambda_{n}}\|p-p\|^{2} \leq g(u)+\frac{1}{2 \lambda_{n}}\|u-p\|^{2}
$$

and hence $J_{\lambda_{n}}^{g} p=p$ for all $n \geq 0$, where $J_{\lambda_{n}}^{g}$ is the Moreau-Yosida resolvent of $g$ in $K$. We have

$$
\left\|u_{n}-p\right\|=\left\|J_{\lambda_{n}}^{g} x_{n}-p\right\| \leq\left\|x_{n}-p\right\|, \quad \forall n \geq 0 .
$$

By Using (3.3) and Lemma 2.7, we have

$$
\begin{aligned}
\left\|y_{n}-p\right\|^{2} & =\left\|\beta_{0}\left(u_{n}-p\right)+\sum_{i=1}^{m} \beta_{i}\left(v_{n}^{i}-p\right)\right\|^{2} \\
& =\beta_{0}\left\|u_{n}-p\right\|^{2}+\sum_{i=1}^{m} \beta_{i}\left\|v_{n}^{i}-p\right\|^{2}-\sum_{i=1}^{m} \beta_{0} \beta_{i}\left\|v_{n}^{i}-u_{n}\right\|^{2}-\sum_{1 \leq i<j}^{m} \beta_{i} \beta_{j}\left\|v_{n}^{i}-v_{n}^{j}\right\|^{2} .
\end{aligned}
$$

Using the fact that, for $i=1, \cdots, m, T_{i} p=\{p\}$, we get

$$
\left\|y_{n}-p\right\|^{2} \leq \beta_{0}\left\|u_{n}-p\right\|^{2}+\sum_{i=1}^{m} \beta_{i}\left(H\left(T_{i} u_{n}, T_{i} p\right)\right)^{2}-\sum_{i=1}^{m} \beta_{0} \beta_{i}\left\|v_{n}^{i}-u_{n}\right\|^{2}-\sum_{1 \leq i<j}^{m} \beta_{i} \beta_{j}\left\|v_{n}^{i}-v_{n}^{j}\right\|^{2} .
$$

Using the fact that, for $i=1, \cdots, m, T_{i}$ is $k_{i}$-strictly pseudo-contractive, we have

$$
\begin{aligned}
\left\|y_{n}-p\right\|^{2} \leq & \beta_{0}\left\|u_{n}-p\right\|^{2}+\sum_{i=1}^{m} \beta_{i}\left(\left\|u_{n}-p\right\|^{2}+k_{i}\left\|v_{n}^{i}-u_{n}\right\|^{2}\right)-\sum_{i=1}^{m} \beta_{0} \beta_{i}\left\|v_{n}^{i}-u_{n}\right\|^{2} \\
& -\sum_{1 \leq i<j}^{m} \beta_{i} \beta_{j}\left\|v_{n}^{i}-v_{n}^{j}\right\|^{2} .
\end{aligned}
$$

Hence,

$$
\left\|y_{n}-p\right\|^{2} \leq\left\|u_{n}-p\right\|^{2}-\sum_{i=1}^{m} \beta_{i}\left(\beta_{0}-k_{i}\right)\left\|v_{n}^{i}-u_{n}\right\|^{2}
$$

Since $\left.\beta_{0} \in\right] \mu, 1[$, we obtain,

$$
\left\|y_{n}-p\right\| \leq\left\|u_{n}-p\right\| \leq\left\|x_{n}-p\right\|
$$

From (3.3), (3.7) and Lemma 2.6, we have

$$
\begin{aligned}
\left\|x_{n+1}-p\right\| & \leq\left\|\left(I-\alpha_{n} \eta A\right) y_{n}-p\right\| \\
& \leq\left(1-\tau \alpha_{n}\right)\left\|x_{n}-p\right\|+\alpha_{n}\|\eta A p\| \\
& \leq \max \left\{\left\|x_{n}-p\right\|, \frac{\|\eta A p\|}{\tau}\right\} .
\end{aligned}
$$

By induction, it is easy to see that

$$
\left\|x_{n}-p\right\| \leq \max \left\{\left\|x_{0}-p\right\|, \frac{\|\eta A p\|}{\tau}\right\}, \quad n \geq 0 .
$$

Hence $\left\{x_{n}\right\}$ is bounded also are $\left.\left\{u_{n}\right)\right\}$, and $\left\{y_{n}\right\}$. 
Consequently, by inequality (3.6) and property of $\mu$, we obtain

$$
\begin{aligned}
\left\|x_{n+1}-p\right\|^{2} & =\left\|P_{K}\left(I-\alpha_{n} \eta A\right) y_{n}-p\right\|^{2} \\
& \leq\left\|y_{n}-p-\alpha_{n} \eta A y_{n}\right\|^{2} \\
& =\left\|y_{n}-p\right\|^{2}+2 \alpha_{n} \eta\left\|y_{n}-p\right\|\left\|A y_{n}\right\|+\alpha_{n}^{2}\left\|\eta A y_{n}\right\|^{2} \\
& \leq\left\|u_{n}-p\right\|^{2}-\sum_{i=1}^{m} \beta_{i}\left(\beta_{0}-k_{i}\right)\left\|v_{n}^{i}-u_{n}\right\|^{2}+2 \alpha_{n} \eta\left\|y_{n}-p\right\|\left\|A y_{n}\right\|+\alpha_{n}^{2}\left\|\eta A y_{n}\right\| \\
& \leq\left\|x_{n}-p\right\|^{2}-\sum_{i=1}^{m} \beta_{i}\left(\beta_{0}-k_{i}\right)\left\|v_{n}^{i}-u_{n}\right\|^{2}+2 \alpha_{n} \eta\left\|y_{n}-p\right\|\left\|A y_{n}\right\|+\alpha_{n}^{2}\left\|\eta A y_{n}\right\| .
\end{aligned}
$$

Thus, for every $i, 1 \leq i \leq m$, we get

$$
\sum_{i=1}^{m} \beta_{i}\left(\beta_{0}-k_{i}\right)\left\|u_{n}^{i}-v_{n}\right\|^{2} \leq\left\|x_{n}-p\right\|^{2}-\left\|x_{n+1}-p\right\|^{2}+2 \alpha_{n} \eta\left\|y_{n}-p\right\|\left\|A y_{n}\right\|+\alpha_{n}^{2}\left\|\eta A y_{n}\right\| .
$$

Since $\left\{x_{n}\right\}$ is bounded, then there exists a constant $B>0$ such that for every $i, 1 \leq i \leq m$,

$$
\sum_{i=1}^{m} \beta_{i}\left(\beta_{0}-k_{i}\right)\left\|v_{n}^{i}-u_{n}\right\|^{2} \leq\left\|x_{n}-p\right\|^{2}-\left\|x_{n+1}-p\right\|^{2}+\alpha_{n} B .
$$

Now we prove that $\left\{x_{n}\right\}$ converges strongly to $x^{*}$. Now we divide the rest of the proof into two cases.

Case 1. Assume that there is $n_{0} \in N$ such that $\left\{\left\|x_{n}-p\right\|\right\}$ is decreasing for all $n \geq n_{0}$. Since $\left\{\left\|x_{n}-p\right\|\right\}$ is monotonic and bounded, $\left\{\left\|x_{n}-p\right\|\right\}$ is convergent. Clearly, we have

$$
\lim _{n \rightarrow \infty}\left[\left\|x_{n}-p\right\|^{2}-\left\|x_{n+1}-p\right\|^{2}\right]=0 .
$$

It then implies from (3.8) that

$$
\lim _{n \rightarrow \infty} \sum_{i=1}^{m} \beta_{i}\left(\beta_{0}-k_{i}\right)\left\|v_{n}^{i}-u_{n}\right\|^{2}=0
$$

Since $\left.\beta_{0} \in\right] \mu, 1[$, we have

$$
\lim _{n \rightarrow \infty}\left\|u_{n}-v_{n}^{i}\right\|^{2}=0
$$

Since $v_{n}^{i} \in T_{i} u_{n}$, it follows that

$$
\lim _{n \rightarrow \infty} d\left(u_{n}, T_{i} u_{n}\right)=0, \forall i=1, \cdots, m .
$$

Let $p \in \Gamma$. Using Lemma 2.9 and since $g(p) \leq g\left(u_{n}\right)$, we get

$$
\left\|x_{n}-u_{n}\right\|^{2} \leq\left\|x_{n}-p\right\|^{2}-\left\|u_{n}-p\right\|^{2} .
$$

Therefore, from (3.3), Lemma 2.2 and inequality (3.13), we get that

$$
\begin{aligned}
\left\|x_{n+1}-p\right\|^{2} & =\left\|\left(I-\alpha_{n} \eta A\right) y_{n}-p\right\|^{2} \\
& \leq\left\|y_{n}-p-\alpha_{n} \eta A y_{n}\right\|^{2} \\
& \leq\left\|y_{n}-p\right\|^{2}+2 \alpha_{n} \eta\left\|y_{n}-p\right\|\left\|A y_{n}\right\|+\alpha_{n}^{2}\left\|\eta A y_{n}\right\| \\
& \leq\left\|u_{n}-p\right\|^{2}+2 \alpha_{n} \eta\left\|y_{n}-p\right\|\left\|A y_{n}\right\|+\alpha_{n}^{2}\left\|\eta A y_{n}\right\|^{2} \\
& \leq\left\|x_{n}-p\right\|^{2}-\left\|x_{n}-u_{n}\right\|^{2}+2 \alpha_{n} \eta\left\|y_{n}-p\right\|\left\|A y_{n}\right\|+\alpha_{n}^{2}\left\|\eta A y_{n}\right\|^{2}
\end{aligned}
$$

and hence

$$
\left\|x_{n}-u_{n}\right\|^{2} \leq\left\|x_{n}-p\right\|^{2}-\left\|x_{n+1}-p\right\|^{2}+2 \alpha_{n} \eta\left\|y_{n}-p\right\|\left\|A y_{n}\right\|+\alpha_{n}^{2}\left\|\eta A y_{n}\right\|^{2} .
$$

Thanks inequality (3.9) and $\alpha_{n} \rightarrow 0$ as $n \rightarrow \infty$, we have

$$
\lim _{n \rightarrow \infty}\left\|x_{n}-u_{n}\right\|=0 .
$$


Next, we prove that $\limsup _{n \rightarrow+\infty}\left\langle x^{*}, x^{*}-x_{n}\right\rangle \leq 0$. Since $H$ is reflexive and $\left\{x_{n}\right\}$ is bounded, there exists a subsequence $\left\{x_{n_{j}}\right\}$ of $\left\{x_{n}\right\}$ such that $x_{n_{j}}$ converges weakly to $\omega$ in $K$ and

$$
\limsup _{n \rightarrow+\infty}\left\langle A x^{*}, x^{*}-x_{n}\right\rangle=\lim _{j \rightarrow+\infty}\left\langle A x^{*}, x^{*}-x_{n_{j}}\right\rangle .
$$

From (3.12) and the fact that $I-T_{i}$ are demiclosed, we obtain $\omega \in \bigcap_{i=1}^{m} F\left(T_{i}\right)$. Using (3.3) and Lemma 2.8 we arrive at

$$
\begin{aligned}
\left\|x_{n}-J_{\lambda}^{g} x_{n}\right\| & \leq\left\|u_{n}-J_{\lambda}^{g} x_{n}\right\|+\left\|u_{n}-x_{n}\right\| \\
& \leq\left\|J_{\lambda_{n}}^{g} x_{n}-J_{\lambda}^{g} x_{n}\right\|+\left\|u_{n}-x_{n}\right\| \\
& \leq\left\|u_{n}-x_{n}\right\|+\left\|J_{\lambda}^{g}\left(\frac{\lambda_{n}-\lambda}{\lambda_{n}} J_{\lambda_{n}}^{g} x_{n}+\frac{\lambda}{\lambda_{n}} x_{n}\right)-J_{\lambda}^{g} x_{n}\right\| \\
& \leq\left\|u_{n}-x_{n}\right\|+\left\|\frac{\lambda_{n}-\lambda}{\lambda_{n}} J_{\lambda_{n}}^{g} x_{n}+\frac{\lambda}{\lambda_{n}} x_{n}-x_{n}\right\| \\
& \leq\left\|u_{n}-x_{n}\right\|+\left(1-\frac{\lambda}{\lambda_{n}}\right)\left\|u_{n}-x_{n}\right\| \\
& \leq\left(2-\frac{\lambda}{\lambda_{n}}\right)\left\|u_{n}-x_{n}\right\| .
\end{aligned}
$$

Hence,

$$
\lim _{n \rightarrow \infty}\left\|x_{n}-J_{\lambda}^{g} x_{n}\right\|=0 .
$$

Since $J_{\lambda}^{g}$ is single valued and nonexpasive, using (3.15) and Lemma 2.1, then $\omega \in F\left(J_{\lambda}^{g}\right)=\operatorname{argmin}_{u \in K} g(u)$. Therefore, $\omega \in \Gamma$. On other hand, using the fact that $x^{*}$ solves (3.4), we then have

$$
\begin{aligned}
\limsup _{n \rightarrow+\infty}\left\langle A x^{*}, x^{*}-x_{n}\right\rangle & =\lim _{j \rightarrow+\infty}\left\langle A x^{*}, x^{*}-x_{n_{j}}\right\rangle \\
& =\left\langle A x^{*}, x^{*}-\omega\right\rangle \leq 0 .
\end{aligned}
$$

Finally, we show that $x_{n} \rightarrow x^{*}$.

$$
\begin{aligned}
\left\|x_{n+1}-x^{*}\right\|^{2}= & \left\|P_{K}\left(I-\eta \alpha_{n} A\right) y_{n}-x^{*}\right\|^{2} \\
\leq & \left\langle\left(I-\eta \alpha_{n} A\right) y_{n}-x^{*}, x_{n+1}-x^{*}\right\rangle \\
= & \left\langle\left(I-\eta \alpha_{n} A\right) y_{n}-x^{*}-\alpha_{n} \eta A x^{*}+\alpha_{n} \eta A x^{*}, x_{n+1}-x^{*}\right\rangle \\
\leq & \left\|\left(I-\alpha_{n} \eta A\right)\left(y_{n}-x^{*}\right)\right\|\left\|x_{n+1}-x^{*}\right\| \\
& +\alpha_{n}\left\langle\eta A x^{*}, x^{*}-x_{n+1}\right\rangle \\
\leq & \left(1-\alpha_{n} \tau\right)\left\|x_{n}-x^{*}\right\|\left\|x_{n+1}-x^{*}\right\|+\alpha_{n}\left\langle\eta A x^{*}, x^{*}-x_{n+1}\right\rangle \\
\leq & \left(1-\alpha_{n} \tau\right)\left\|x_{n}-x^{*}\right\|^{2}+2 \alpha_{n} \eta\left\langle A x^{*}, x^{*}-x_{n+1}\right\rangle .
\end{aligned}
$$

From Lemma 2.3, its follows that $x_{n} \rightarrow x^{*}$. We can check that all the assumptions of Lemma 2.3 are satisfied. Therefore, we deduce $x_{n} \rightarrow x^{*}$.

Case 2. Assume that there is not $n_{0} \in \mathbb{N}$ such that $\left\{\left\|x_{n}-x^{*}\right\|\right\}$ is not monotonically decreasing sequence. Set $\Omega_{n}=\left\|x_{n}-x^{*}\right\|$ and $\tau: \mathbb{N} \rightarrow \mathbb{N}$ be a mapping for all $n \geq n_{0}$ (for some $n_{0}$ large enough) by $\tau(n)=\max \{k \in \mathbb{N}: k \leq$ $\left.n, \Omega_{k} \leq \Omega_{k+1}\right\}$.

We have $\tau$ is a non-decreasing sequence such that $\tau(n) \rightarrow \infty$ as $n \rightarrow \infty$ and $\Omega_{\tau(n)} \leq \Omega_{\tau(n)+1}$ for $n \geq n_{0}$. From (3.8), we have

$$
\sum_{i=1}^{m} \beta_{i}\left(\beta_{0}-k_{i}\right)\left\|u_{\tau(n)}-v_{\tau(n)}^{i}\right\|^{2} \leq \alpha_{\tau(n)} B .
$$

Furthermore, we have

$$
\lim _{n \rightarrow+\infty} \sum_{i=1}^{m} \beta_{i}\left(\beta_{0}-k_{i}\right)\left\|u_{\tau(n)}-v_{\tau(n)}^{i}\right\|^{2}=0 .
$$


Since $\left.\beta_{0} \in\right] \mu, 1[$, we have

$$
\lim _{n \rightarrow \infty}\left\|u_{\tau(n)}-v_{\tau(n)}^{i}\right\|^{2}=0
$$

Since $v_{\tau(n)}^{i} \in T_{i} u_{\tau(n)}$, it follows that

$$
\lim _{n \rightarrow \infty} d\left(u_{\tau(n)}, T_{i} u_{\tau(n)}\right)=0 \forall i=1, \cdots, m .
$$

By same argument as in case 1 , we can show that $x_{\tau(n)}$ converges weakly in $K$ and $\limsup _{n \rightarrow+\infty}\left\langle A x^{*}, x^{*}-x_{\tau(n)}\right\rangle \leq 0$. We have for all $n \geq n_{0}$,

$$
0 \leq\left\|x_{\tau(n)+1}-x^{*}\right\|^{2}-\left\|x_{\tau(n)}-x^{*}\right\|^{2} \leq \alpha_{\tau(n)}\left[-\tau\left\|x_{\tau(n)}-x^{*}\right\|^{2}+2 \eta\left\langle A x^{*}, x^{*}-x_{\tau(n)+1}\right\rangle\right],
$$

which implies that

$$
\left\|x_{\tau(n)}-x^{*}\right\|^{2} \leq \frac{2 \eta}{\tau}\left\langle A x^{*}, x^{*}-x_{\tau(n)+1}\right\rangle .
$$

Then, we have

$$
\lim _{n \rightarrow \infty}\left\|x_{\tau(n)}-x^{*}\right\|^{2}=0
$$

Therefore,

$$
\lim _{n \rightarrow \infty} B_{\tau(n)}=\lim _{n \rightarrow \infty} B_{\tau(n)+1}=0 .
$$

Thus, by Lemma 2.4, we conclude that

$$
0 \leq B_{n} \leq \max \left\{B_{\tau(n)}, B_{\tau(n)+1}\right\}=B_{\tau(n)+1} .
$$

Hence, $\lim _{n \rightarrow \infty} B_{n}=0$, that is $\left\{x_{n}\right\}$ converges strongly to $x^{*}$. This completes the proof.

Now, we apply Algorithm 1 for solving fixed points problem involving multivalued nonexpansive mappings and convex minimization problem without demiclosedness assumption.

Theorem 3.2. Let $H$ be a real Hilbert space and $K$ be a nonempty, closed convex cone of $H$. Let $A: K \rightarrow H$ be an $\alpha$-strongly monotone and L-Lipschitzian operator, $m \geq 1$ be a fixed number, for $i, 1 \leq i \leq m$, let $T_{i}: K \rightarrow C B(K)$ be a multivalued $k_{i}$-strictly pseudo-contractive mapping and $g: K \rightarrow(-\infty,+\infty)$ be a proper convex and lower semi-continuous function such that $\Gamma:=\bigcap_{i=1}^{m} F\left(T_{i}\right) \cap \operatorname{argmin}_{y \in K} g(y) \neq \emptyset$. Let $\left\{x_{n}\right\}$ be a sequence defined iteratively from arbitrary $x_{0} \in K$ by:

$$
\left\{\begin{array}{l}
u_{n}=\operatorname{argmin}_{u \in K}\left[g(u)+\frac{1}{2 \lambda_{n}}\left\|u-x_{n}\right\|^{2}\right] \\
y_{n}=\beta_{0} u_{n}+\sum_{i=1}^{m} \beta_{i} v_{n}^{i}, \quad v_{n}^{i} \in T_{i} u_{n} \\
x_{n+1}=P_{K}\left(I-\alpha_{n} \eta A\right) y_{n}, \quad n \geq 0 .
\end{array}\right.
$$

With conditions $\left\{\alpha_{n}\right\} \subset(0,1)$ and $\eta>0$ satisfy:

(i) $\lim _{n \rightarrow \infty} \alpha_{n}=0, \quad(i i) 0<\eta<\frac{2 \alpha}{L^{2}}$ and $\sum_{n=0}^{\infty} \alpha_{n}=\infty$,

(iii) $\left.\beta_{0} \in\right] \mu, 1\left[, \quad \mu:=\max \left\{k_{i}, \quad i=1, \ldots, m\right\}, \quad \beta_{i} \in\right] 0,1\left[\right.$ and $\beta_{0}+\beta_{1}+\cdots \beta_{m}=1$.

(iv) $\left\{\lambda_{n}\right\}$ is a sequence such that $\lambda_{n} \geq \lambda>0$ for all $n \geq 1$ and some $\lambda$. Then, the sequences $\left\{x_{n}\right\}$ and $\left\{u_{n}\right\}$ defined by Algorithm 1 converge strongly to $x^{*} \in \Gamma$, which is a minimizer of $g$ in $K$ as well as it is also a common fixed points of $T_{i}$ in $K$.

Proof. Since every multivalued nonexpansive mapping is multivalued strictly pseudo-contractive mapping, then, the proof follows Lemma 2.1 and Theorem 3.1. 
Corollary 3.1. Let $H$ be a real Hilbert space. Let $m \geq 1$ be a fixed number, for $i, 1 \leq i \leq m$, let $T_{i}: H \rightarrow H$ be a $k_{i}$-strictly pseudo-contractive mapping and $g: H \rightarrow(-\infty,+\infty)$ be a proper convex and lower semi-continuous function such that $\Gamma:=\bigcap_{i=1}^{m} F\left(T_{i}\right) \cap \operatorname{argmin}_{y \in B} g(y) \neq \emptyset$. Let $\left\{x_{n}\right\}$ be a sequence defined iteratively from arbitrary $x_{0} \in H$ by:

$$
\left\{\begin{array}{l}
u_{n}=\operatorname{argmin}_{u \in H}\left[g(u)+\frac{1}{2 \lambda_{n}}\left\|u-x_{n}\right\|^{2}\right], \\
y_{n}=\beta_{0} u_{n}+\sum_{i=1}^{m} \beta_{i} T_{i} u_{n} \\
x_{n+1}=\left(1-\alpha_{n}\right) y_{n}, \quad n \geq 0 .
\end{array}\right.
$$

With conditions $\left\{\alpha_{n}\right\} \subset(0,1)$ satisfies:

(i) $\lim _{n \rightarrow \infty} \alpha_{n}=0, \quad($ ii $) \sum_{n=0}^{\infty} \alpha_{n}=\infty$,

(iii) $\left.\beta_{0} \in\right] \mu, 1\left[, \quad \mu:=\max \left\{k_{i}, \quad i=1, \ldots, m\right\}, \quad \beta_{i} \in\right] 0,1\left[\right.$ and $\beta_{0}+\beta_{1}+\cdots \beta_{m}=1$.

(iv) $\left\{\lambda_{n}\right\}$ is a sequence such that $\lambda_{n} \geq \lambda>0$ for all $n \geq 1$ and some $\lambda$. Then, the sequences $\left\{x_{n}\right\}$ and $\left\{u_{n}\right\}$ defined by Algorithm 1 converge strongly to $x^{*} \in \Gamma$.

Proof. Since every single-valued strictly pseudo-contractive is multivalued strictly pseudo-contractive mapping, then, the proof follows Theorem 3.1.

\section{Conclusion}

The problem of finding a common element of the set of fixed points of nonlinear operators and the set of solutions of convex minimization problem has attracted much attention because of its extraordinary utility and broad applicability in many branches of mathematical science and engineering. General terative algorithm and proximal point algorithm are remarkably useful methods for solving most important problems with nonlinear operators. In this article, we introduce and analyze a new iterative algorithm for approximating a common solution of an equilibrium problem, variational inequality problems and fixed point problems with a finite family of multivalued strictly pseudo-contractive mappings without imposing any compactness-type condition on either the operators or the space considered. The results obtained in this paper are important improvements of recent important results in this field.

\section{References}

[1] L. Ambrosio, N. Gigli, G. Savaré, Gradient flows in metric spaces and in the space of probability measures, Second edition, Lectures in Mathematics ETH Zürich, Birkhäuser Verlag, Basel, (2008).

[2] Berinde, V., Pcurar, M., The role of the Pompeiu-Hausdorff metric in fixed point theory. Creat. Math. Inform. 22 (2013), no. 2, 143-150.

[3] F.S. Blasi, J. Myjak, S. Reich, A.J Zaslavski, Generic existence and approximation of fixed points for nonexpansive set-valued maps, Set-Valued Var. Anal. 17(1), 97-112 (2009).

[4] F. E. Browder, Convergenge theorem for sequence of nonlinear operator in Banach spaces, Math.Z. 100 (1967). 201-225. Vol. EVIII, part 2, 1976.

[5] F. E. Browder, and W. V. Petryshyn, Construction of fixed points of nonlinear mappings in Hilbert spaces, J. Math. Anal. Appl. 20 (1967) 197- 228.

[6] C. E. Chidume, Geometric Properties of Banach spaces and Nonlinear Iterations, Springer Verlag Series: Lecture Notes in Mathematics, Vol. 1965,(2009), ISBN 978-1-84882-189-7.

[7] C.E. Chidume, N. Djitte, Iterative algorithm for zeros of bounded m-Accretive nonlinear operators, to appear, J. Nonlinear and convex analysis.

[8] C.E. Chidume, N. Djitte, Strong convergence theorems for zeros of bounded maximal monotone nonlinear operators, J. Abstract and Applied Analysis, Volume 2012, Article ID 681348, 19 pages, doi:10.1155/2012/681348. 
[9] C. E. Chidume, N. Djitté, M. Sène, Iterative algorithm for zeros of multi-valued accretive operators in certain Banach spaces, Afr. Mat. 26 (2015), no. 3-4: 357-368.

[10] C. E. Chidume, C. O. Chidume, N. Djitte, and M. S. Minjibir, Krasnoselskii-type algorithm for fixed points of multi-valued strictly pseudo-contractive, Fixed Point Theory and Applications 2013, 2013:58.

[11] J. Jost, Convex functionals and generalized harmonic maps into spaces of nonpositive curvature, Comment. Math. Helv., 70 (1995), 659-673.

[12] O. Güler, On the convergence of the proximal point algorithm for convex minimization, SIAM J. Control Optim., 29 (1991), 403-419.

[13] S. Kakutani, A generalization of Brouwer's fixed point theorem, Duke Mathematical Journal 8 (1941), no. 3, 457-459.

[14] S. Kamimura, W. Takahashi, Strong convergence of a proximal-type algorithm in a Banach space, SIAM J. of Optimization 13(3) (5003), 938-945.

[15] N. Lehdili, A. Moudafi, Combining the proximal algorithm and Tikhonov regularization, Optimization 37(1996), 239-252.

[16] W.R. Mann, Mean value methods in iteration, Proc. Amer. Math. Soc., 4 (1953) 506-510.

[17] P. E. Mainge, Strong convergence of projected subgradient methods for nonsmooth and nonstrictly convex minimization, Set-Valued Analysis, 16, 899-912 (2008).

[18] G. Marino and H. K. Xu, A general iterative method for nonexpansive mappings in Hibert spaces, J. Math. Anal. Appl. 318 (2006), 43-52.

[19] G. Marino, H.K. Xu, Weak and strong convergence theorems for strict pseudo-contractions in Hilbert spaces, J. Math. Math. Appl., 329(2007), 336-346.

[20] Moudafi, A: Viscosity approximation methods for fixed point problems. J. Math. Anal. Appl. 241, 46-55 (2000).

[21] B. Martinet, Régularisation d'inéquations variationnelles par approximations successives, (French) Rev. Franaise Informat. Recherche Opérationnelle, 4 (1970), 154-158.

[22] A. A. Mebawondu, Proximal point algorithms for finding common fixed points of a finite family of nonexpansive mapping of nonexpansive multivalued mappings in real Hilbert spaces, Khayyam J. Math. 5 (2019) no. 2, 113-123.

[23] I. Miyadera, Nonlinear semigroups, Translations of Mathematical Monographs, American Mathematical Society, Providence, (1992).

[24] J.F. Nash, Equilibrium points in n-person games, Proceedings of the National Academy of Sciences of the United States of America, 36 (1950), no1, 48-49.

[25] J.F. Nash, Non-coperative games, Annals of Mathematics, Second series 54 (1951), 286-295.

[26] B. Panyanak, Ishikawa iteration processes for multi-valued mappings in Banach Spaces, Comput. Math. Appl. 54 (2007), 872-877.

[27] J. Garcia-Falset, E. Lorens-Fuster, and T. Suzuki, Fixed point theory for a classs of generalised nonexpansive mappings, J. Math. Anal. Appl. 375 (2011), 185-195.

[28] S. Reich, Strong Convergence theorems for resolvents of accretive operators in Banach spaces, in J. Math. Anal. Appl. 183 (1994), 118-120.

[29] R.T. Rockafellar, On the maximality of sums of nonlinear monotone operators. Trans. Am.Math. Soc. 149, 7588 (1970).

[30] M. Sene, P. Faye and N. Djitté, A Krasnoselskii type Algorithm approximating a common Fixed Point of a finite family of multivalued strictly pseudo-contractive mappings in Hilbert spaces ,J. Maths. Sci. Adv. Appl.,Volume 27, 2014, Pages 59-80. 
[31] M.V. Solodov, B.F. Svaiter, Forcing strong convergence of proximal point iterations in a Hilber space, Math. Program., Ser. A 87 (5000) 189-202.

[32] T.M.M. Sow, A new iterative method for multivalued nonexpansive mappings in Banach spaces with application Journal Nonlinear Analysis and Application 2018 No.2 (2018) 212-222.

[33] T.M.M. Sow, N. Djitté, and C.E. Chidume, A path convergence theorem and construction of fixed points for nonexpansive mappings in certain Banach spaces, Carpathian J.Math.,32(2016),No.2,217-226,2016.

[34] T. M. M. Sow, M. Sène, N. Djitté, Strong convergence theorems for a common fixed point of a finite family of multi-valued Mappings in certain Banach Spaces, Int. J. Math. Anal., Vol. 9, 2015, no. 9, 437-452.

[35] H.K. Xu, An iterative approach to quadratic optimization, J. Optim. Theory Appl. 116 (2003) 659-678.

[36] H.K. Xu, Iterative algorithms for nonlinear operators, J. London Math. Soc. 66 (2002), no. 2, 240 - 256.

[37] Y. Yao, H. Zhou, Y. C. Liou, Strong convergence of modified Krasnoselskii-Mann iterative algorithm for nonexpansive mappings, J. Math. Anal. Appl. Comput. 29 (2009) 383-389.

[38] S. Wang, A general iterative method for an infinite family of strictly pseudo-contractive mappings in Hilbert spaces, Applied Mathematics Letters, 24(2011): 901-907.

\section{Affiliations}

THIERNO M. M. SOW

ADDRESS: Department of Mathematics, Gaston Berger University, Saint Louis, Senegal

E-MAIL: sowthierno89@gmail.com

ORCID ID: 0000-0002-9687-839X 